\section{RSP}

http://www.rsp.fsp.usp.br/
Revista de Saúde Pública

\title{
Satisfação dos usuários em relação aos serviços de Atenção Primária à Saúde no Brasil
}

\author{
Juliana Leal Ribeiro Cantalino' iD, Magda Duarte dos Anjos Scherer' iD, Jacks Soratto" \\ Antônio Augusto Schäfer" (iD, Davllyn Santos Oliveira dos Anjos' (iD) \\ ' Universidade de Brasília. Faculdade de Ciências da Saúde. Programa de Pós-Graduação em Saúde Coletiva. \\ Brasília, DF, Brasil \\ " Universidade do Extremo Sul Catarinense. Programa de Pós-Graduação em Saúde Coletiva. Criciúma, SC, \\ Brasil
}

\section{RESUMO}

OBJETIVO: Analisar a satisfação de usuários em relação ao acesso, à infraestrutura e à qualidade dos serviços na Atenção Primária à Saúde (APS) no Brasil.

MÉTODOS: Estudo transversal com dados de 114.615 usuários vinculados a 30.523 equipes de saúde, obtidos por meio do banco de dados do Programa Nacional de Melhoria do Acesso e da Qualidade da Atenção Básica (PMAQ-AB). Foram estudadas variáveis independentes relacionadas ao acesso, à infraestrutura e à qualidade dos serviços na APS. O desfecho, satisfação dos usuários, foi mensurado através das variáveis: "se tivesse a opção, mudaria de equipe ou de serviço de saúde" e "recomendaria este serviço de saúde para um amigo ou familiar". Para avaliar a satisfação de acordo com as variáveis independentes de exposição, foi utilizado o teste Qui-quadrado de Pearson, considerando nível de significância de 5\%. Análises descritivas das variáveis foram realizadas através de frequências absoluta (n) e relativa (\%).

Correspondência:

Juliana Leal Ribeiro Cantalino Faculdade de Ciências da Saúde

Campus Universitário Darcy

Ribeiro, s/n

Asa Norte 70910-900. Brasília, DF

E-mail: juliana.Irc@gmail.com

Recebido: 7 fev 2020

Aprovado: 21 jul 2020

Como citar: Cantalino JLR, Schere MDA, Soratto I, Schäfer AA, Anjos DSO. Satisfação dos usuários em relação aos serviços de Atenção Primária à Saúde no Brasil. Rev Saude Publica. 2021;55:22. https://doi.org/10.11606/s15188787.2021055002533

Copyright: Este é um artigo de acesso aberto distribuído sob os termos da Licença de Atribuição Creative Commons, que permite uso irrestrito, distribuição e reprodução em qualquer meio desde que o autor e a fonte originais sejam creditados.
RESULTADOS: A satisfação dos usuários esteve associada às variáveis de acesso ( $p<0,001)$ infraestrutura $(\mathrm{p}<0,001)$ e qualidade dos serviços $(\mathrm{p}<0,001)$ na APS. A proximidade do serviço, atenção à demanda espontânea, escuta e o respeito dos profissionais às singularidades do paciente, assim como a resolutividade dos serviços, sem necessidade de encaminhamentos para outros e a boa infraestrutura, estiveram relacionados com a satisfação dos usuários.

CONCLUSÃO: Para a garantia da melhoria da qualidade dos serviços ofertados na APS do Brasil, os aspectos de satisfação dos usuários identificados no presente estudo devem ser considerados na organização e gestão dos serviços.

DESCRITORES: Atenção Primária à Saúde. Satisfação do Paciente. Avaliação em Saúde. Serviços de Saúde. 


\section{INTRODUÇÃO}

A satisfação dos usuários com os serviços deve ser considerada na avaliação e no planejamento das ações de Atenção Primária à Saúde (APS). A satisfação perpassa vários fatores, entre os quais se encontram a garantia de acesso aos serviços quando necessário, a qualidade do atendimento prestado pelos profissionais e as condições estruturais dos locais que concedem assistência ${ }^{1,2}$. A satisfação do usuário é algo complexo e difícil de avaliar pois tem dimensão subjetiva e forte relação com as características do processo de trabalho.

A APS constitui importante estratégia internacional dos sistemas universais de saúde. No Brasil, foi materializada por uma série de ações de saúde individuais ou coletivas de promoção, prevenção, diagnóstico, redução de danos e vigilância em saúde, realizada por equipes multiprofissionais que atuam em território adstrito ${ }^{3}$.

A expansão da APS nos últimos anos, com cobertura populacional em torno de $74 \%$ no território nacional ${ }^{4}$, tem colaborado para redução dos indicadores de morbimortalidade, com contribuições significativas na redução da mortalidade infantil e nas internações por causas sensíveis à atenção primária ${ }^{5}$. Entretanto, o acesso ainda é muito heterogêneo, sendo por vezes precário ${ }^{6}$, causando a insatisfação dos usuários ${ }^{7}$ e indicando uma situação que desafia profissionais, gestores e pesquisadores a repensarem ações para sua melhoria. O acesso é a capacidade da APS de acolher os usuários promovendo a vinculação com os profissionais e corresponsabilização pela atenção às suas necessidades de saúde. Atuar na perspectiva de melhora do acesso significa contribuir para qualidade da atenção à saúde .

A qualidade dos serviços tem sido alvo de queixas dos usuários, uma vez que a demora para agendamento de consultas e a não assistência à demanda contínua que surge nos serviços constituem os principais fatores para insatisfação dos usuários com a APS ${ }^{8}$. Além disso, a infraestrutura da APS é outro aspecto que merece atenção, afinal, estrutura dos serviços deve levar em conta os preceitos da ambiência, de modo a proporcionar uma atenção acolhedora e humana para os usuários, além de um ambiente confortável para o trabalho dos profissionais de saúde ${ }^{3}$. Mesmo com incentivo financeiro do Ministério da Saúde para requalificação dos serviços de saúde ${ }^{9}$, ainda há muito a avançar, tendo em vista que muitos ainda apresentam situações precárias também para os profissionais, com fragilidades na estrutura física, organizacional e falta de materiais e insumos ${ }^{10-13}$.

Diante do exposto, o presente estudo tem por objetivo analisar a satisfação de usuários em relação ao acesso, à infraestrutura e à qualidade dos serviços na APS no Brasil.

\section{MÉTODOS}

Trata-se de um estudo transversal com dados secundários obtidos por meio do banco de dados nacional da avaliação externa do $2^{\circ}$ Ciclo do Programa Nacional de Melhoria do Acesso e da Qualidade da Atenção Básica (PMAQ-AB), realizado no ano de 2014¹4. O PMAQ-AB é um programa de remuneração por desempenho do Ministério da Saúde que tem como objetivo principal incentivar a ampliação do acesso e a melhoria da qualidade da atenção básica, como é denominada a atenção primária no Brasil, com o intuito de estabelecer um padrão de qualidade comparável nacional, regional e localmente. Através de ferramentas de avaliação e disponibilização dos dados coletados, o PMAQ-AB possibilita transparência e efetividade das ações governamentais nesse âmbito de atuação ${ }^{14}$.

O Programa foi concebido em quatro fases complementares: 1) adesão ao programa; 2) implantação de dispositivos de reorganização dos processos de trabalho da equipe e da gestão (autoavaliação, educação permanente, apoio institucional e monitoramento); 3) avaliação externa, feita por especialistas e por usuários; 4) pactuação com o incremento de padrões e indicadores de qualidade ${ }^{14}$. 
Para avaliação externa foram aplicados seis instrumentos com objetivos diferentes, sendo que, neste estudo, foram utilizadas variáveis do módulo 3, que se refere à entrevista para avaliação da satisfação dos usuários com os serviços de Atenção Primária ofertados pelas Unidades Básicas de Saúde (UBS) ${ }^{15}$.

A aplicação do módulo 3 ocorreu por meio de entrevista estruturada que buscou conhecer as características sociodemográficas dos participantes e a satisfação em relação aos serviços ofertados. Os usuários foram selecionados de forma aleatória, sendo quatro por UBS avaliada, com os seguintes critérios de inclusão: usuários que não passaram por consulta com médico, enfermeiro ou dentista no dia da entrevista; com idade acima de 18 anos; que não estivessem realizando a primeira consulta; e que não estavam retornando depois de ausência de mais de 12 meses em relação à data da entrevista. Quando o usuário selecionado não atendia aos critérios, outro era selecionado aleatoriamente, não tendo sido registradas recusas.

Ressaltamos que o PMAQ é realizado numa parceria entre o Ministério da Saúde e 41 instituições de ensino e pesquisa, que realizaram treinamento exaustivo com o núcleo de entrevistadores e supervisores responsáveis pela coleta dos dados a fim de proporcionar uma padronização nacional dos dados com o objetivo de melhorar a qualidade dos registros. O instrumento de coleta de dados, por ser realizado via aplicativo instalado em tablets, possuía um conjunto de regras de validação em sua programação, que realizava a validação interna das respostas enquanto o instrumento era preenchido. Em uma terceira etapa, os dados eram validados pelo sistema do Ministério da Saúde com o objetivo de avaliar a completude dos questionários e os dados de identificação das equipes entrevistadas. Somente após essas etapas foi realizada a certificação das equipes e a disponibilização dos bancos de dados para o acesso público dos pesquisadores.

A população esperada era de 122.092 usuários, entretanto, foram registrados 7.477 (6,12\%) perdas por ausência de usuário nos serviços de APS para responder ao questionário no momento da coleta de dados ou por serem de equipes que desistiram de serem avaliadas. Ao final, foram incluídos 114.615 usuários das 30.523 equipes de saúde da atenção primária do Brasil, abrangendo 5.057 (90,8\%) municípios do Brasil que fizeram parte do $2^{\circ}$ ciclo do PMAQ-AB.

Para o presente estudo, foram selecionadas pelos pesquisadores 7,2\% das 319 variáveis do módulo 3, totalizando 23 variáveis. Dessas, 17 são variáveis independentes relacionadas ao acesso, à infraestrutura e à qualidade dos serviços na APS (descritas nas tabelas 2, $3 \mathrm{e}$ 4). Para fins de análise, essas variáveis foram dicotomizadas quando apresentavam mais de duas categorias de resposta. O desfecho, satisfação dos usuários, foi mensurado através de duas variáveis: "se tivesse a opção, mudaria de equipe ou de serviço de saúde" (sim/não) e "recomendaria este serviço de saúde para um amigo ou familiar" (sim/não). Também foram incluídas as variáveis sociodemográficas: sexo (masculino, feminino), faixa etária ( $\leq 29,30-54,55-64, \geq 65)$, cor da pele (branca, preta, parda/mestiça, amarela, indígena), escolaridade (não é alfabetizado [não sabe ler e escrever], é alfabetizado [sabe ler e escrever], ensino fundamental incompleto, ensino fundamental completo, ensino médio incompleto, ensino médio completo, ensino superior incompleto, ensino superior completo), a fim de caracterizar a população.

A análise dos dados foi realizada através do software estatístico IBM SPSS versão 20.0. Para avaliar a satisfação dos usuários de acordo com o acesso, a infraestrutura e a qualidade dos serviços na APS, foi utilizado o teste Qui-quadrado de Pearson, considerando nível de significância de $5 \%$. Análises descritivas das variáveis foram realizadas através de frequências absoluta (n) e relativa (\%).

\section{RESULTADOS}

A maioria dos entrevistados era do sexo feminino (79,6\%), predominando a faixa etária entre 30 e 54 anos (44,4\%), raça/cor da pele autodeclarada parda/mestiça $(46,2 \%)$ e com ensino fundamental incompleto $(36,0 \%)$ (Tabela 1$)$. 
A Tabela 2 mostra a relação entre acesso e satisfação dos usuários. Pode-se observar que os indivíduos que recomendariam o serviço de saúde a um amigo ou familiar e que não mudariam de equipe ou serviço de saúde são aqueles que responderam que o tempo de

Tabela 1. Características sociodemográficas dos usuários estudados. Brasil, 2014 ( $\mathrm{n}=114.615)$.

\begin{tabular}{lcc}
\hline Características & $\mathrm{n}$ & $\%$ \\
\hline Sexo & 23.412 & 20,4 \\
\hline Masculino & 91.203 & 79,6 \\
\hline Feminino & & \\
\hline Faixa etária (anos) & 27.479 & 24,0 \\
\hline$\leq 29$ & 50.899 & 44,4 \\
\hline $30-54$ & 18.771 & 16,4 \\
\hline $55-64$ & 17.466 & 15,2 \\
\hline$\geq 65$ & & \\
\hline Raça/Cor da pele (n=112.472) & & 36,6 \\
\hline Branca & 41.145 & 13,3 \\
\hline Preta & 15.010 & 46,2 \\
\hline Parda/mestiça & 51.909 & 3,1 \\
\hline Amarela & 3.528 & 0,8 \\
\hline Indígena & 880 & \\
\hline Escolaridade & & 7,6 \\
\hline Não é alfabetizado (não sabe ler e escrever) & 8.669 & 7,4 \\
\hline É alfabetizado (sabe ler e escrever) & 8.491 & 36,0 \\
\hline Ensino fundamental incompleto & 41.213 & 10,6 \\
\hline Ensino fundamental completo & 12.169 & 10,5 \\
\hline Ensino médio incompleto & 11.966 & 21,7 \\
\hline Ensino médio completo & 24.840 \\
\hline Ensino superior incompleto & 3.078 \\
\hline Ensino superior completo & 4.045 & 2,7 \\
\hline Percentuá & & 3,5 \\
\hline
\end{tabular}

a Percentual máximo de observações desconhecidas para a variável raça/cor da pele: 2.143 (1,9\%).

Tabela 2. Satisfação de usuários em relação ao acesso a serviços de atenção primária à saúde. Brasil, 2014 ( $\mathrm{n}=114.615)$.

\begin{tabular}{|c|c|c|c|c|c|c|c|c|c|c|c|}
\hline & \multirow{3}{*}{ n total } & \multicolumn{5}{|c|}{$\begin{array}{c}\text { Se tivesse a opção, mudaria de equipe ou serviço } \\
\text { de saúde }\end{array}$} & \multicolumn{5}{|c|}{$\begin{array}{c}\text { Recomendaria o serviço de saúde para um amigo } \\
\text { ou familiar }\end{array}$} \\
\hline & & \multicolumn{2}{|c|}{ Sim } & \multicolumn{2}{|c|}{ Não } & \multirow{2}{*}{ p-valor } & \multicolumn{2}{|c|}{ Sim } & \multicolumn{2}{|c|}{ Não } & \multirow{2}{*}{$\mathbf{p}$} \\
\hline & & $\mathbf{n}$ & $\%$ & $\mathbf{n}$ & $\%$ & & $\mathbf{n}$ & $\%$ & $\mathbf{n}$ & $\%$ & \\
\hline $\begin{array}{l}\text { Tempo de deslocamento do } \\
\text { domicílio ao Serviço de Saúde }\end{array}$ & 114.195 & & & & & & & & & & \\
\hline$<$ de 30 minutos & & 15.072 & 16,0 & 79.398 & 84,0 & $<0,001$ & 81.973 & 86,8 & 12.497 & 13,2 & $<0,001$ \\
\hline$\geq$ a 30 minutos & & 5.093 & 25,8 & 14.632 & 74,2 & & 16.297 & 82,6 & 3.428 & 17,4 & \\
\hline $\begin{array}{l}\text { Facilidade de acesso ao } \\
\text { Serviço de Saúde }\end{array}$ & 114.615 & & & & & & & & & & \\
\hline Muito fácil/fácil & & 13.568 & 14,7 & 78.523 & 85,3 & $<0,001$ & 80.541 & 87,5 & 11.550 & 12,5 & $<0,001$ \\
\hline Razoável/difícil e muito difícil & & 6.668 & 29,6 & 15.856 & 70,4 & & 18.076 & 80,3 & 4.448 & 19,7 & \\
\hline $\begin{array}{l}\text { Horário de funcionamento do } \\
\text { serviço de saúde atende às } \\
\text { necessidades }\end{array}$ & 113.076 & & & & & & & & & & \\
\hline Sim & & 13374 & 13,6 & 84.824 & 86,4 & $<0,001$ & 87.976 & 89,6 & 10.222 & 10,4 & $<0,001$ \\
\hline Não & & 6450 & 43,4 & 8.428 & 56,6 & & 9.509 & 63,9 & 5.369 & 36,1 & \\
\hline $\begin{array}{l}\text { Qualidade do atendimento } \\
\text { quando não tem horário } \\
\text { agendado }\end{array}$ & 67.277 & & & & & & & & & & \\
\hline Muito boa/boa & & 6717 & 12,1 & 48.983 & 87,9 & $<0,001$ & 50.810 & 91,2 & 4.890 & 8,8 & $<0,001$ \\
\hline Regular/ruim/muito ruim & & 5723 & 49,4 & 5.854 & 50,6 & & 7.225 & 62,4 & 4.352 & 37,6 & \\
\hline
\end{tabular}


deslocamento da sua residência ao serviço de saúde foi menor do que 30 minutos $(\mathrm{p}<0,001)$, que consideraram muito fácil ou fácil o acesso ao serviço de saúde ( $<<0,001)$, que referiram que o horário de funcionamento do serviço de saúde atende às suas necessidades $(\mathrm{p}<0,001)$ e que consideraram muito boa ou boa a qualidade do atendimento quando não se tem horário agendado $(\mathrm{p}<0,001)$.

Quanto à qualidade do atendimento e à satisfação dos usuários estudados (Tabela 3), os resultados indicam que os indivíduos que recomendariam o serviço de saúde a um

Tabela 3. Satisfação de usuários em relação à qualidade do atendimento em serviços de atenção primária à saúde. Brasil, 2014 (n = 114.615).

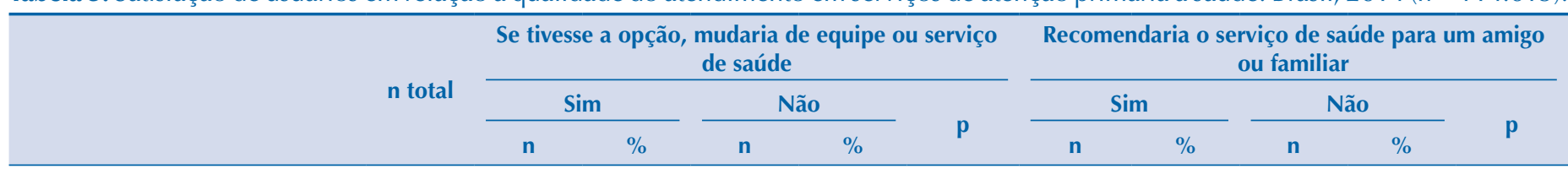

A equipe busca resolver as necessidades/problemas no próprio Serviço de Saúde

\section{Sempre/na maioria das vezes}

Quase nunca/nunca

Consultório para atendimento é um lugar reservado, tem privacidade

$$
\text { Sim }
$$$$
\text { Não }
$$

Os profissionais fornecem orientações sobre necessidade de repouso, alimentação adequada e como tomar os remédios

\section{Sempre/na maioria das vezes \\ Quase nunca/nunca}

Sente-se respeitado(a) pelos profissionais em relação aos hábitos culturais, costumes, religião

$$
\text { Sempre/na maioria das vezes }
$$$$
\text { Quase nunca/nunca }
$$

Tempo de consulta com o médico é suficiente

Nim

Tempo de consulta com o enfermeiro é suficiente

$$
\text { Sim }
$$

Tem facilidade para falar com os profissionais que lhe atenderam para tirar dúvidas após consultas

$$
\text { Sempre/na maioria das vezes }
$$

Quase nunca/nunca

Encontra facilidade para conversar com os profissionais sobre os resultados dos exames

$$
\text { Sempre/na maioria das vezes }
$$

Quase nunca/nunca

Qualidade do cuidado recebido pela equipe de saúde
113.185

$\begin{array}{cccccccccc}13.887 & 13,7 & 87.799 & 86,3 & <0,001 & 91.122 & 89,6 & 10.564 & 10,4 & <0,001 \\ 6.037 & 52,5 & 5.462 & 47,5 & & 6.362 & 55,3 & 5.137 & 44,7 & \end{array}$

113.859

$\begin{array}{cccccccccc}17.338 & 16,1 & 90.375 & 83,9 & <0,001 & 94.181 & 87,4 & 13.532 & 12,6 & <0,001 \\ 2.713 & 44,1 & 3.433 & 55,9 & & 3.868 & 62,9 & 2.278 & 37,1 & \end{array}$

113.409

$\begin{array}{cccccccccc}14.864 & 14,7 & 86.072 & 85,3 & <0,001 & 89.614 & 88,8 & 11.322 & 11,2 & <0,001 \\ 5.114 & 41,0 & 7.359 & 59,0 & & 8.046 & 64,5 & 4.427 & 35,5 & \end{array}$

113.777

$\begin{array}{cccccccccc}17.905 & 16,3 & 92.057 & 83,7 & <0,001 & 96.133 & 87,4 & 13.829 & 12,6 & <0,001 \\ 2079 & 54,5 & 1.736 & 45,5 & & 1.896 & 49,7 & 1.919 & 50,3 & \end{array}$

112.372

$\begin{array}{llllllllll}11.864 & 12,7 & 81.275 & 87,3 & <0,001 & 83.616 & 89,8 & 9.523 & 10,2 & <0,001 \\ 7.854 & 40,8 & 11.379 & 59,2 & & 13.230 & 68,8 & 6.003 & 31,2 & \end{array}$

103.789

$\begin{array}{cccccccccc}13.525 & 14,3 & 81.281 & 85,7 & <0,001 & 84.159 & 88,8 & 10.647 & 11,2 & <0,001 \\ 4.082 & 45,4 & 4.901 & 54,6 & & 5.689 & 63,3 & 3.294 & 36,7 & \end{array}$

79.216

$\begin{array}{cccccccccc}8.062 & 12,3 & 57.404 & 87,7 & <0,001 & 59.452 & 90,8 & 6.014 & 9,2 & <0,001 \\ 6.073 & 44,2 & 7.677 & 55,8 & & 8.766 & 63,8 & 4.984 & 36,2 & \end{array}$

105.586

$\begin{array}{llllllllll}11.089 & 13,0 & 74.363 & 87,0 & <0,001 & 76.967 & 90,1 & 8.485 & 9,9 & <0,001 \\ 7.424 & 36,9 & 12.710 & 63,1 & & 14.022 & 69,6 & 6.112 & 30,4 & \end{array}$

114.378

\begin{tabular}{lcccccccccc} 
Muito boa/boa & 9.381 & 10,0 & 84.876 & 90,0 & $<0,001$ & 87.284 & 92,6 & 6.973 & 7,4 & $<0,001$ \\
\hline Regular/ruim/muito ruim & 10.780 & 53,6 & 9.341 & 46,4 & & 11.205 & 55,7 & 8.916 & 44,3 & \\
\hline
\end{tabular}


Tabela 4. Satisfação de usuários em relação à infraestrutura de serviços de atenção primária à saúde. Brasil, 2014 (n = 114.615).

\begin{tabular}{|c|c|c|c|c|c|c|c|c|c|c|}
\hline & \multicolumn{5}{|c|}{$\begin{array}{c}\text { Se tivesse a opção, mudaria de equipe ou serviço de } \\
\text { saúde }\end{array}$} & \multicolumn{5}{|c|}{$\begin{array}{c}\text { Recomendaria o serviço de saúde para um amigo ou } \\
\text { familiar }\end{array}$} \\
\hline n total & \multicolumn{2}{|c|}{ Sim } & \multicolumn{2}{|c|}{ Não } & \multirow[b]{2}{*}{$\mathbf{p}$} & \multicolumn{2}{|c|}{ Sim } & \multicolumn{2}{|c|}{ Não } & \multirow[b]{2}{*}{ p } \\
\hline & $\mathbf{n}$ & $\%$ & $\mathbf{n}$ & $\%$ & & $\mathrm{n}$ & $\%$ & $n$ & $\%$ & \\
\hline
\end{tabular}

As instalações do serviço de saúde estão em boas condições de uso

Sim

114.615

$11,9 \quad 75.038$

$34,2 \quad 19.341$

$88,1<0,001$

65,8

\subsection{1}

21.376

90,7

$90,7 \quad 7.961$

$72,7 \quad 8.037$

$<0,001$

As instalações do serviço de saúde estão em boas condições de limpeza

$$
\text { Sim }
$$

Não

Quantidade suficiente de cadeiras para as pessoas sentarem no local de espera

Sim

Percepção sobre as instalações do serviço de saúde

Muito boa/boa
Regular/ruim/muito ruim

114.615

$\begin{array}{cccccccccc}13.526 & 13,8 & 84.460 & 86,2 & <0,001 & 87.562 & 89,4 & 10.424 & 10,6 & <0,001 \\ 6710 & 40,4 & 9.919 & 59,6 & & 11.055 & 66,5 & 5.574 & 33,5 & \end{array}$

114.615

\begin{tabular}{cccccccccc}
6265 & 10,2 & 55.390 & 89,8 & $<0,001$ & 56.449 & 91,6 & 5.206 & 8,4 & $<0,001$ \\
13971 & 26,4 & 38.989 & 73,6 & & 42.168 & 79,6 & 10.792 & 20,4 & \\
& & & & & & & & & \\
& & & & & & & & & \\
6278 & 8,6 & 66.958 & 91,4 & $<0,001$ & 67.891 & 92,7 & 5.345 & 7,3 & $<0,001$ \\
13860 & 33,9 & 26.975 & 66,1 & & 30.297 & 74,2 & 10.538 & 25,8 & \\
\hline
\end{tabular}

amigo ou familiar e que não mudariam de equipe ou serviço de saúde eram aqueles que consideraram que sempre - ou na maioria das vezes - a equipe buscava resolver as suas necessidades/problemas no próprio serviço de saúde ( $\mathrm{p}<0,001)$. Além disso, também consideraram que o consultório para atendimento é um lugar reservado, que proporciona privacidade durante a consulta $(\mathrm{p}<0,001)$, que sempre - ou na maioria das vezes - os profissionais fornecem orientações sobre necessidade de repouso, alimentação adequada e como tomar os remédios ( $\mathrm{p}<0,001)$, assim como sentem-se respeitados(as) pelos profissionais em relação aos hábitos culturais, costumes e religião $(p<0,001)$. Consideraram também que o tempo de consulta com o médico e enfermeiro é suficiente ( $p<0,001)$, que sempre - ou na maioria das vezes - têm facilidade para falar com os profissionais que lhes atenderam para tirar dúvidas após consultas ( $\mathrm{p}<0,001)$, da mesma forma que encontram facilidade para conversar com os profissionais sobre os resultados dos exames $(\mathrm{p}<0,001)$ e se referiram à qualidade do cuidado recebido pela equipe de saúde como muito boa e boa $(\mathrm{p}<0,001)$.

Com relação à associação entre a infraestrutura e satisfação dos usuários (Tabela 4), evidenciou-se que os indivíduos que recomendariam o serviço de saúde a um amigo ou familiar e que não mudariam de equipe ou serviço foram aqueles que referiram: boas condições de uso e limpeza das instalações do serviço de saúde $(\mathrm{p}<0,001)$; quantidade suficiente de cadeiras para as pessoas sentarem no local de espera $(\mathrm{p}<0,001)$; muito boa ou boa percepção sobre as instalações do serviço de saúde $(\mathrm{p}<0,001)$.

\section{DISCUSSÃO}

Importante achado do presente estudo é que a maioria dos usuários entrevistados referiu satisfação em relação ao acesso, à infraestrutura e à qualidade dos serviços na APS no Brasil.

A distância entre o serviço de saúde e o domicílio desempenhou um significativo papel na satisfação, corroborando com os resultados da avaliação do $1^{\circ}$ Ciclo do PMAQ-AB ${ }^{16}$. A satisfação pode estar relacionada com a possibilidade de não usar meio de transporte para chegar ao serviço e com o curto tempo necessário para realizar o percurso ${ }^{17}$. 
Análises de dados do PMAQ-AB de 2012 em Pernambuco evidenciaram que a maioria dos usuários $(73,2 \%)$ considerava que os serviços de saúde se encontravam próximos de seus domicílios, algo já esperado tendo em vista que os serviços de saúde com menor complexidade costumam estar mais próximos da população ${ }^{18}$. Entretanto, a simples proximidade, por mais que seja um aspecto importante a influenciar na satisfação dos usuários, não garante a real utilização.

Em relação à satisfação dos usuários quanto ao horário de funcionamento, diversos trabalhos realizados no âmbito da APS, corroborando com o presente estudo, encontraram resultados positivos ${ }^{17-19}$. O funcionamento do serviço de APS em no mínimo cinco dias na semana, com carga horária de, pelo menos, oito horas, com disponibilidade de medicamentos e resultados de exames, bem como consultas com especialistas asseguradas, além de maior quantidade de unidades de saúde no município, foram identificados como fatores que tornam o serviço mais acessível e, dessa forma, satisfatório para os usuários ${ }^{17-19}$.

Entretanto, os resultados da pesquisa sobre o $1^{\circ}$ ciclo, realizado entre 2012 e 2013, evidenciaram que o funcionamento da APS, apenas em horário comercial, não atende às necessidades da população entrevistada nas cinco grandes regiões brasileiras ${ }^{16}$. O fechamento dos serviços de APS por determinado período para a realização de reuniões das equipes, preconizado como essencial pelas diretrizes nacionais para o trabalho na APS, contribui para a insatisfação de usuários com relação ao horário de funcionamento dos serviços ${ }^{17}$.

Outro item importante de satisfação dos usuários em relação ao acesso é o atendimento à demanda espontânea. Observa-se que, entre os ciclos 1 e 2 do PMAQ-AB, as reservas de vagas para atendimento no mesmo dia passaram de $64,9 \%$ para $88,1 \%$, sinalizando melhora neste indicador ${ }^{20}$. Dos usuários que procuram os serviços de APS para algum atendimento imediato, a maioria relata ter conseguido a consulta ${ }^{19}$.

Os resultados encontrados referentes à facilidade de acesso aos serviços pela maioria dos usuários entrevistados coincidem com o encontrado na avaliação do $1^{\circ}$ ciclo do PMAQ-AB, na qual $83 \%$ das equipes da APS foram classificadas como acessíveis para os usuários ${ }^{19}$. Condições técnico-assistencial, econômica, política e simbólica são constituintes do conceito de acesso aos serviços de saúde ${ }^{7}$, entretanto, essas condições podem ser afetadas por questões como localização, população e características socioeconômicas, que podem influenciar o acesso ao serviço de APS $^{21}$. Alguns atributos da APS demonstraram melhora entre os ciclos 1 e 2 do PMAQ, como a integralidade e o primeiro contato ${ }^{20}$. Tais atributos podem ter influenciado o nível de satisfação dos usuários com o serviço e colaborado para redução nas desigualdades no acesso e utilização dos serviços de saúde do Brasil ${ }^{22}$.

Quanto ao alto percentual de usuários que consideraram que os profissionais e os serviços de saúde se apresentam acessíveis e resolutivos às suas necessidades quando procuram os serviços de APS, esse desfecho positivo pode ter sido produzido pela fragilidade no processo de seleção dos entrevistados, visto que o mesmo só levou em consideração a opinião do grupo que conseguiu acesso ao atendimento, sendo este um dos fatores limitadores desse estudo.

As fragilidades existentes na rede de coordenação do cuidado da APS influenciam no percentual de resolutividade dos problemas de saúde dos usuários ${ }^{23}$. Se a correta captação da necessidade do usuário puder ser realizada e coordenada pela atenção primária, porta de entrada do Sistema Único de Saúde (SUS), o percentual de satisfação quanto ao serviço pode ser aumentado, o que os leva a indicar os serviços de APS para seus familiares e conhecidos $^{24}$. Isso pode ser decorrente do fato de que o usuário não precisa percorrer longos caminhos na rede especializada ou alta complexidade.

Quanto à existência de consultório para atendimento com privacidade ao paciente, os resultados encontrados ratificam estudos que identificaram que a satisfação do usuário diminui $41 \%$ quando o consultório não está em uma área reservada ${ }^{25}$, seja para troca de roupa 
ou realização de exames ${ }^{26}$. A falta de privacidade tem uma dimensão ética, afeta a dignidade humana, dificulta a escuta, o desenvolvimento do vínculo e da confiança na relação entre usuário e profissional. Esses aspectos têm relação com o tempo que o profissional médico ou enfermeiro despende com o paciente, que também tem associação significativa com a satisfação dos usuários dos serviços ${ }^{1,27,28}$.

Em relação ao respeito aos hábitos culturais, costumes e religião, os indivíduos que se sentem respeitados pelos profissionais têm forte influência na avaliação positiva dos serviços de saúde ${ }^{1,25}$, como evidenciado pelo presente estudo e pelo de Protasio et al. ${ }^{25}$ Esse aspecto de satisfação pode estar associado aos modos de produção do cuidado, que quando orientados pela "valorização dos saberes, da experiência e da autonomia dos usuários, pelo entendimento do outro como ser semelhante, tendem a contribuir para ações mais criativas e que qualificam o cuidado na APS"29.

Em relação à infraestrutura dos serviços de APS, houve predominância de avaliação positiva das instalações pelos usuários. Condições políticas, organizacionais e estruturais que garantem a execução de funções essenciais das Unidades de Saúde é condição para maior satisfação dos usuários e desafio para construção de uma APS forte e abrangente ${ }^{30}$.

O estudo demonstrou que a satisfação dos usuários está associada a aspectos elementares para funcionamento dos serviços de APS, que dependem do compromisso dos gestores para com os espaços de realização do cuidado, que devem ter boas instalações físicas, condições salubres de higiene e limpeza, mobiliário, materiais e insumos suficientes para o exercício das práticas profissionais.

A redução da satisfação dos usuários às dimensões do acesso, qualidade do atendimento e infraestrutura dos serviços, assim como a dificuldade em conceituar o termo qualidade na avaliação de um serviço, trazem limitações ao presente estudo. Entretanto, as variáveis selecionadas para avaliar a satisfação permitiram traçar um panorama de como os usuários percebem os serviços da APS. A utilização de dados secundários, de uma forma geral, poderia se constituir numa limitação ao estudo, entretanto, os dados secundários utilizados no presente estudo são de um banco de dados público proveniente de uma pesquisa nacional a qual seguiu os critérios de padronização de coleta a fim de proporcionar qualidade dos registros.

Os elementos de satisfação dos usuários em relação ao acesso, qualidade e infraestrutura dos serviços de saúde ratificam a importância de se garantir, cada vez mais, que as pessoas tenham condições não só de acessar os serviços de saúde, como também sejam atendidas em locais com estruturas físicas adequadas e com profissionais capacitados a lidar com as diferenças e singularidades.

Os usuários recomendariam e não mudariam de equipe se o tempo de deslocamento ao serviço fosse curto, houvesse facilidade para acessá-lo, bom horário de funcionamento, qualidade do atendimento à demanda espontânea, resolução dos problemas no próprio serviço, existência de orientações em saúde, respeito às diversidades, tempo suficiente de consulta, facilidade para sanar dúvidas diversas e sobre exames, qualidade do cuidado recebido, e boa infraestrutura.

Nessa perspectiva, para a garantia da melhoria da qualidade dos serviços ofertados pelos serviços de APS no Brasil, os aspectos de satisfação dos usuários identificados no presente estudo devem ser considerados na organização e gestão dos serviços.

\section{REFERÊNCIAS}

1. Moimaz SAS, Marques JAM, Saliba O, Garbin CAS, Zina LG, Saliba NA. Satisfação e percepção do usuário do SUS sobre o serviço público de saúde. Physis. 2010;20(4):1419-40. https://doi.org/10.1590/S0103-73312010000400019 
2. Arruda CAM, Bosi MLM. Satisfação de usuários da atenção primária à saúde: um estudo qualitativo no Nordeste do Brasil. Interface (Botucatu). 2017;21(61):321-32. https://doi.org/10.1590/1807-57622015.0479

3. Brasil. Portaria № 2.436, de 21 de setembro de 2017. Aprova a Política Nacional de Atenção Básica, estabelecendo a revisão de diretrizes para a organização da Atenção Básica, no âmbito do Sistema Único de Saúde (SUS). Diário Oficial União [Internet]. 22 set. 2017. Disponível em: http://bvsms.saude.gov.br/bvs/saudelegis/gm/2017/prt2436_22_09_2017.html

4. Secretaria de Atenção Primária à Saúde. Cobertura da Atenção Básica [Internet]. Brasília, DF: Ministério da Saúde; 2017 [citado em 25 set. 2019]. Disponível em: https://egestorab.saude.gov. br/paginas/acessoPublico/relatorios/relHistoricoCoberturaAB.xhtml

5. Facchini LA, Tomasi E, Dilélio AS. Qualidade da atenção primária à saúde no Brasil: avanços, desafios e perspectivas. Saúde Debate. 2018;42(Espec 1):208-23. https://doi.org/10.1590/0103-11042018s114

6. Tesser $\mathrm{CD}$, Norman AH, Vidal TB. Acesso ao cuidado na Atenção Primária à Saúde brasileira: situação, problemas e estratégias de superação. Saúde Debate. 2018;42(Espec 1):361-78. https://doi.org/10.1590/0103-11042018s125

7. Paula WKAS, Samico IC, Caminha MFC, Batista Filho M, Silva SL. Avaliação da atenção básica à saúde sob a ótica dos usuários: uma revisão sistemática. Rev Esc Enferm USP. 2016;50(2):33545. https://doi.org/10.1590/S0080-623420160000200021

8. Gomide MFS, Pinto IC, Bulgarelli AF, Santos ALP, Gallardo MPS. A satisfação do usuário com a atenção primária à saúde: uma análise do acesso e acolhimento. Interface (Botucatu). 2018;22(65):387-98. https://doi.org/10.1590/1807-57622016.0633

9. Bousquat A, Giovanella L, Fausto MCR, Fusaro ER, Mendonça MHM, Gagno J et al. Tipologia da estrutura das unidades básicas de saúde brasileiras: os 5 R. Cad Saúde Pública. 2017;33(8). https://doi.org/10.1590/0102-311×00037316

10. Munyewende PO, Rispel LC, Chirwa T. Positive practice environments influence job satisfaction of primary health care clinic nursing managers in two South African provinces. Hum Resour Health. 2014;12:27. https://doi.org/10.1186/1478-4491-12-27

11. Tambasco LP, Silva HS, Pinheiro KMK, Gutierrez BAO. A satisfação no trabalho da equipe multiprofissional que atua na Atenção Primária à Saúde. Saúde Debate. 2017;41(Espec 2):140-51. https://doi.org/10.1590/0103-11042017s212

12. Moura BLA, Cunha RC, Fonseca ACF, Aquino R, Medina MG, Vilasbôas ALQ et al. Atenção primária à saúde: estrutura das unidades como componente da atenção à saúde. Rev Bras Saúde Matern Infant. 2010;10(Supl 1):S69-81 . https://doi.org/10.1590/S1519-38292010000500007

13. Soratto J, Pires DEP, Trindade LL, Oliveira JSA, Forte ECN, Melo TP. Insatisfação no trabalho de profissionais da saúde na estratégia saúde da família. Texto \& Contexto Enferm. 2017;26(3):e2500016. https://doi.org/10.1590/0104-07072017002500016

14. Programa de Melhoria do Acesso e da Qualidade. Manual instrutivo do Pmaq para as equipes de Atenção Básica (Saúde da Família, Saúde Bucal e Equipes Parametrizadas) e NASF. 2. ed. Brasília, DF: Ministério da Saúde; 2015. 66 p.

15. Programa de Melhoria do Acesso e da Qualidade. Instrumento de avaliação externa para as equipes de Atenção Básica (saúde da família e equipe parametrizada). Brasília, DF: Ministério da Saúde; 2013. 129 p.

16. Protasio APL, Gomes LB, Machado LS, Valença AMG. Satisfação do usuário da atenção básica em saúde por regiões do Brasil: $1^{\circ}$ ciclo de avaliação externa do PMAQ-AB. Ciênc Saude Coletiva. 2017;22(6):1829-44. https://doi.org/10.1590/1413-81232017226.26472015

17. Santos SMS, Oliveira VAC, Oliveira RAC, Guimarães EAA. Estratégia saúde da família: qualidade da assistência sob a perspectiva da satisfação do usuário. Rev Min Enferm. 2010;14(4):499-508.

18. Albuquerque MSV, Lyra TM, Farias SF, Medeiros MF, Martelli MPJL. Acessibilidade aos serviços de saúde: uma análise a partir da Atenção Básica em Pernambuco. Saúde Debate. 2014;38(Espec):182-94. https://doi.org/10.5935/0103-1104.2014S014

19. Fausto MCR, Giovanella L, Mendonça MHM, Fonseca HMS, Lima JG. A posição da Estratégia Saúde da Família na rede de atenção à saúde na perspectiva das equipes e usuários participantes do PMAQ-AB 2012. Saúde Debate. 2014;38(Espec):13-33. https://doi.org/10.5935/0103-1104.2014S003 
20. Lima JG, Giovanella L, Fausto MCR, Bousquat A, Silva EV. Atributos essenciais da Atenção Primária à Saúde: resultados nacionais do PMAQ-AB. Saúde Debate. 2018;42(Espec 1):52-66. https://doi.org/10.1590/0103-11042018s104

21. Alves MGM, Casotti E, Oliveira LGD, Machado MTC, Almeida PF, Corvino MPF et al. Fatores condicionantes do acesso às equipes da Estratégia Saúde da Família no Brasil. Saúde Debate. 2014;38(Espec):34-51. https://doi.org/10.5935/0103-1104.2014S004

22. Macinko J, Lima-Costa MF. Horizontal equity in health care utilization in Brazil, 1998-2008. Int J Equity Health. 2012;11(1):1-8. https://doi.org/10.1186/1475-9276-11-33

23. Hollanda E, Siqueira SAV, Andrade GRB, Molinaro A, Vaitsman J. Satisfação e responsividade em serviços de atenção à saúde da Fundação Oswaldo Cruz. Ciênc Saúde Coletiva. 2012;17(12):3343-52. https://doi.org/10.1590/S1413-81232012001200019

24. Rodrigues LBB, Silva PCS, Peruhype RC, Palha PF, Popolin MP, Crispim JA et al. A atenção primária à saúde na coordenação das redes de atenção: uma revisão integrativa. Ciênc Saúde Coletiva. 2014;19(2):343-52. https://doi.org/10.1590/1413-81232014192.18032012

25. Rosa RB, Pelegrini AHW, Lima MADS. Resolutividade da assistência e satisfação de usuários da estratégia saúde da família. Rev Gaúcha Enferm. 2011;32(2):345-51. https://doi.org/10.1590/S1983-14472011000200019

26. Protasio APL, Gomes LB, Machado LS, Valença AMG. Fatores associados à satisfação de usuários com a atenção primária à saúde no Brasil. Cad Saúde Pública. 2017;33(2). https://doi.org/10.1590/0102-311x00184715

27. Scherer M, Menezes E. Un espace potentiel de créativité : les soins primaires de santé. Education Permanente. 2015;202:91-100.

28. Aldana JM, Piechulek $\mathrm{H}, \mathrm{Al}$-Sabir A. Client satisfaction and quality of health care in rural Bangladesh. Bull World Health Organ. 2001;79(6):512-7.

29. Bastos GAN, Fasolo LR. Fatores que influenciam a satisfação do paciente ambulatorial em uma população de baixa renda: um estudo de base populacional. Rev Bras Epidemiol. 2013;16(1):114-24. https://doi.org/10.1590/S1415-790X2013000100011

30. Bousquat A, Giovanella L, Fausto MCR, Medina MG, Martins CL, Almeida PF et al. A atenção primária em regiões de saúde: política, estrutura e organização. Cad Saúde Pública. 2019;35(Supl 2):e00099118. https://doi.org/10.1590/0102-311x00099118

Financiamento: DSOA recebeu bolsa do PDSE da Coordenação de Aperfeiçoamento de Pessoal de Nível Superior - Brasil (Capes código de Financiamento 001).

Contribuição dos Autores: Concepção e planejamento do estudo; coleta, análise e interpretação dos dados; elaboração e revisão do manuscrito; aprovação da versão final; e responsabilidade pública pelo seu conteúdo: JLRC, MDAS, JS, AAS, DSOA.

Conflito de Interesses: Os autores declaram não haver conflito de interesses. 